\title{
A Network Coding Based Routing Protocol in Wireless Sensor Networks
}

\author{
Min Zhu ${ }^{1,2}$, Dengyin Zhang ${ }^{1}$, Zhanxiang Ye $^{2}$, Xuemei Wang ${ }^{3}$ and Jin Wang ${ }^{4}$ \\ ${ }^{1}$ Internet of Things Technology Park, Nanjing University of Posts and \\ Telecommunications, Nanjing, 210003, China \\ ${ }^{2}$ Information \& Technical Department, Wenzhou Vocational \& Technical \\ College, Wenzhou 325000 \\ ${ }^{3}$ College of Computer, Nanjing University of Posts and Telecommunications, \\ Nanjing, 210003, China \\ ${ }^{4}$ College of Information Engineering, Yangzhou University, Yangzhou 225009, \\ China
}

\begin{abstract}
Since wireless sensor networks have limited energy resources, energy efficiency and reliable data transmission rate are key issues in designing the topology. In this paper, a new root protocol NCQ-DD is proposed in order to solve the issue of limited performance of $D D$ in wireless sensor networks. NCQ-DD greatly improves traditional DD protocol by utilizing random linear network coding technology and service quality perception. Experimental results show that the new routing protocol can save network bandwidth resources and sensor node's energy, improve data transmission rate and ensure reliable transmission, thus increasing the lifetime of sensor networks.
\end{abstract}

Keywords: Wireless Sensor Network, network coding, service quality awareness

\section{Introduction}

WSN (Wireless Sensor Network) is widely applied in various fields with terrific performance, such as in the public healthy service, agricultural technology and environment monitoring. According to the complex requirement of environment monitoring and the limited energy of each node, reducing power consumption and reliable transmission is vital in the research of wireless senor network [1].

In terms of the implementation methodology of routing protocols, there are majorly four sorts of routing protocols: the routing protocols based on cluster, such as LEACH[2], TEEN[3], PEGASIS[4]; the routing protocols based on data, such as SPIN[5], DD[6], RUMOR [7]; the routing protocols based on geographic position, such as SPAN[8], GEAR[9], GRWLI; the routing protocols based on power awareness, such as GAF[10] and EQR[11]. Furthermore, in the routing protocols based on cluster head selection, the random selection of cluster head nodes and asymmetric distribution of cluster head nodes in the process of data integration, the individual cluster head node can decrease lifetime of whole network. In the geography based routing protocols, certain extra routing consumption can be generated due to collection of geographic positions. The energy awareness based routing protocols are not suitable for large scale network. DD routing protocol proposes the idea of directed diffusion and data centralization, which is the major researching tendency and the important cornerstone of WSN design [12]. Data centralized routing protocol can effectively reduce the redundancy of information transmission and prolong the lifetime of network. However, building data structure still needs time and power consumption. The highlight of this paper is the improvement of directed diffusion routing protocol by the technique of random linear-network coding and the idea of service 
quality awareness.

\section{Directed Diffusion Routing Protocol}

Directed Diffusion Routing Protocol (DD) is one of typical routing protocol in WSN. It is driven by data and query to realize the process of interest diffusion, gradient construction and routes enhancement.

In the step of interest diffusion, sink nodes generate the interest message, according to the different requirements from different applications. Then, sink nodes flood this interest message to other nodes and inform the message information to all the sensor nodes. Since the interest message is sent by flooding, each node can receive the same interest message from different neighbor nodes. The current nodes assign the interest message with different gradient number.

In the period of gradient construction, the data gradient from source node to sink node is generated by routes message which is recorded during the period of interest diffusion. Specifically, once the source node which contains the information required by the sink node receives the interest diffusion message, it sends detecting data to sink node with low speed through the same direction of gradient. After that, the detecting data from different source nodes can arrive the sink node successively and the sink node selects the best route which is the shortest path as the transmission route.

In the period of routes enhancement, once the sink node receives data from source node, the enhanced route will be generated between sink node and source node. After this route is built, data between source node and sink node can be transmitted with higher speed. Compared with the speed in the detecting period, the performance of speed in this period is improved dramatically.

Whereas the drawback of DD protocol is that it rely on the flooding mechanism in the interest diffusion period and the broadcasting transmission in the gradient construction period, leading to huge consumption of time and power. Moreover, in the routes enhancement period, there is only one route between each source node and sink node. Thus, when this route becomes invalid, another route should be searched. The robustness is decreased, wasting of resources and average delay is increased. DD protocol merely takes delay into consideration to select best routes, neglecting energy consumption of selecting routes and energy exhaustion of nodes in the network. Therefore it is easy to generate the unbalanced energy allocation of the whole network and the exhausted nodes.

\section{NCQ-DD Based on Network Coding and Service Awareness}

According to these drawback mentioned above, this paper addresses NCQ-DD( Network Coding and QoS-Aware based Directed Diffusion Protocol ), one new wireless sensor network routing protocol, which is an improved DD routing protocol with the technique of random linear-network coding and the idea of service quality awareness.

\subsection{Improvement Strategy of Protocol}

The transmission nodes encode the received message by linear network coding and non-linear network coding and transmit the message to next hop. Under the consideration of the remaining energy of nodes and valid bandwidth, the best path is selected based on the measured delay in various routes.

In the period of routes construction, when source node sends detecting message, the nodes on the corresponding routes record the minimum remaining energy of nodes and the total energy consumption in the message. This information can return to the source node. Assuming $\mathrm{Elj}$ is the minimum remaining energy of each node. Elj=min (Esource,........Esink). Etj is the total energy consumption on the jth route to transmit this message, then the energy level of each node on each route is 
$\mathrm{A}$ and $\mathrm{B}$ is equal to 1 and 2 respectively as the optimal values in this paper, which are the weight.

The range of one-hop transmission is regarded as the conflicted area. If there is communication in this area, the bandwidth of other nodes can be influenced. The valid bandwidth of node $\mathrm{n}$ is

$$
B R_{n}=B W-\sum_{i \in I(n)} B D_{i}
$$

BW is the initial bandwidth of each node. I(n) is the set of neighbor nodes with one-hop. BDi is the occupied bandwidth in i and BRn denotes the available bandwidth in node $\mathrm{n}$. The valid bandwidth for each route is

$$
\mathrm{B}_{\mathrm{j}}=\min \left\{\mathrm{BR}_{\mathrm{n}}\right\}, \mathrm{n} \in E(j)
$$

$E(j)$ denotes the set of all the nodes on route $j$.

The level of each valid route with consideration of energyof node and remaining bandwidth is levelj $=$ Enerjm $*$ Bjn (4). In this equation, $\mathrm{m}$ and $\mathrm{n}$ denote the weight of Enerj and $\mathrm{Bj}$ respectively. In this paper, the optimal values are 3 and 2 respectively for $\mathrm{m}$ and $\mathrm{n}$. The optimal route with the maximum level value is selected to transmit data.

Assuming $\mathrm{h}$ denotes the number of the routes between sinks and source node in certain time. The enhancement route is selected from the routes which reach $h / 2$ routes in the shortest time. When the optimal route is selected, the routes which take the second place is saved as the backup. If the optimal route is invalid, the backup can work immediately without routing search.

\subsection{Description of Routing Process}

The steps of the new routing protocol NCQ-DD are as below.

Step 1 is the period of interest diffusion which is to search information required source nodes.

(a). Assuming maximum capacity of network is $\mathrm{h}$, the data packet is divided into $\mathrm{h}$ sets of vectors: B1, B2......Bh, and generates $m$ sets of random vectors $(m>h)$. There are $h$ random vectors $(\mathrm{r} 1, \ldots \ldots, \mathrm{rh})$ in each set. An encoding packet can be generated by the vectors in the set times one set of random vectors: $E 1=r 1 B 1+\ldots \ldots . r h B h . ~ a=(r 1, \ldots \ldots, r h)$ is the global coding vectors. The number of $\mathrm{m}$ encoding packets is generated by this methodology. The coding packets and corresponding global coding vectors are sent to the neighbor nodes in the form (E,a). For the source node, the global coding vector is unit vector.

(b). When the intermediate node receives the coding packets, it can judge whether to receive the packets for the first time. If the packets have been received, the new arrivals are discarded. Otherwise, the intermediate node can determine whether it is the destination node of interest diffusion message. If it is, the packets are saved and the procedure jumps into step 3 . If not, the packets are saved and local coding vectors with h-dimensionality are generated at random in the GF( $2 h$ ) finite field, which can encode the number of $h$ data message and transmit the new (E,a) to next hop. Assuming a1, a2,......ah is the coding packets received by intermediate node and $\mathrm{E} 1, \mathrm{E} 2, \ldots . . . \mathrm{Eh}$ is the original global coding vectors respectively. $(\mathrm{b} 1, \mathrm{~b} 2, \ldots \ldots ., \mathrm{bh})$ is the local coding vectors generated at random. A and $\mathrm{E}$ are new global coding vectors and coding packets respectively generated by intermediate node. $\mathrm{A}=\mathrm{b} 1 \mathrm{a} 1+\mathrm{b} 2 \mathrm{a} 2+\ldots . . . \mathrm{bhah}, \mathrm{E}=\mathrm{b} 1 \mathrm{E} 1+\mathrm{b} 2 \mathrm{E} 2+\ldots . . . \mathrm{bhEh}$.

(c). When the source node receives coding packets, it can judge whether the number of received coding packets $n$ is greater than or equal to $h(n>=h)$. If not, the intermediate node keeps waiting for the new coding packets. If it is, the received packets are decoded by Gauss Elimination to get the original interest diffusion packets. The process of decoding is as follows: 


$$
\left[\begin{array}{l}
E_{1} \\
E_{2} \\
\vdots \\
E_{h}
\end{array}\right]=\left(\begin{array}{lll}
r_{1}^{1} & \cdots & r_{h}^{1} \\
\vdots & \ddots & \vdots \\
r_{1}^{h} & \cdots & r_{h}^{h}
\end{array}\right)\left[\begin{array}{c}
B_{1} \\
B_{2} \\
\vdots \\
B_{h}
\end{array}\right] \Rightarrow\left[\begin{array}{l}
B_{1} \\
B_{2} \\
\vdots \\
B_{h}
\end{array}\right]=\left(\begin{array}{lll}
r_{1}^{1} & \cdots & r_{h}^{1} \\
\vdots & \ddots & \vdots \\
r_{1}^{h} & \cdots & r_{h}^{h}
\end{array}\right)^{-1}\left[\begin{array}{l}
E_{1} \\
E_{2} \\
\vdots \\
E_{h}
\end{array}\right]
$$

$\mathrm{E}$ denotes the number of $\mathrm{h}$ data vectors received by destination node and $\mathrm{r}$ denotes the global coding vectors. When the finite field is up to 216 and transmission routes are less than 28 , the randomly generated local coding vectors are linearly independent and the hit rate of decoding is almost $100 \%$.

Step 2 is the period of routes construction to determine the available routes between source nodes and sink node.

(a). The coding process for detecting data is similar to the coding process of interest diffusion. However, there are two additional records in the source node: the minimum remaining energy value of node in this route $\mathrm{El}$ and the total energy consumption of data packets transmission Et, setting the initial value as -1 .

(b). When the intermediate node receives the data detecting coding packets, it can judge whether to receive the packets for the first time. If the packets have been received, the new arrivals are discarded. Otherwise, the intermediate node can determine whether it is the destination node of coding packets. If it is, the packets are saved and the procedure jumps into step 3. If not, the packets are saved and local coding vectors with h-dimensionality are generated at random in the GF( $2 \mathrm{~h})$ finite field, which can encode the number of $h$ data message and acquire the new $(E, a)$. At the same time, the energy required by this node is added into Et. Compared the energy of this node with $\mathrm{El}$, if $\mathrm{El}$ is greater, then the node updates $\mathrm{El}$ and transmits new coding packets to the next hop.

(c). When the source node receives coding packets, it can judge whether the number of received coding packets $n$ is greater than or equal to $h(n>=h)$. If not, the intermediate node keeps waiting for the new coding packets. If it is, the received packets are decoded by Gauss Elimination to get the original packets. The process of decoding is similar to the process of decoding in the period of interest diffusion.

Step 3 is the period of routes enhancement.

Parameter $\mathrm{h}$ denotes the number of the valid routes between sink and source node. The level values of the $h / 2$ routes with the minimum delay are calculated by formula (4). Furthermore, remaining energy is calculated by formula (1) and remaining valid bandwidth is Figured out by formula (2) and (3). The route with the largest value of level is selected as the optimal route. When the optimal route is selected, the route which takes the second place is saved as the backup. Other routing information is removed.

Step 4 is the period of data transmission.

All the data is transmitted through the optimal route between source node and sink node. When the transmission is not completed, if the current route becomes invalid, then the node checks out whether there is backup route. If it is, the backup route becomes the optimal route. If not, the process of interest diffusion restarts to search the valid routes.

\section{Simulation}

In this paper, the properties of DD protocol and NCQ-DD protocol are compared in the aspects of packet arrival rate, end-to-end delay and average remaining energy of nodes. The major experimental parameters are shown in this table. 
Table 1. Configuration of Experimental Parameters

\begin{tabular}{|c|c|}
\hline Bandwidth & $2 \mathrm{Mbps}$ \\
\hline Field & $800 \mathrm{~m} * 800 \mathrm{~m}$ \\
\hline MAC Layer & IEEE $802.11 \mathrm{DCF}$ \\
\hline Data Rate & $6^{*} 64 \mathrm{bit} / \mathrm{s}$ \\
\hline Number of Nodes & $10 \mathrm{~J}$ \\
\hline Initial Energy & $0.660 \mathrm{~J}$ \\
\hline $\begin{array}{c}\text { Sending } \\
\text { Consumption } \\
\text { Receiving } \\
\text { Consumption }\end{array}$ & $0.395 \mathrm{~J}$ \\
\hline $\begin{array}{c}\text { Sleeping } \\
\text { Consumption }\end{array}$ & $0.035 \mathrm{~J}$ \\
\hline
\end{tabular}

(1) Packet Arrival Rate

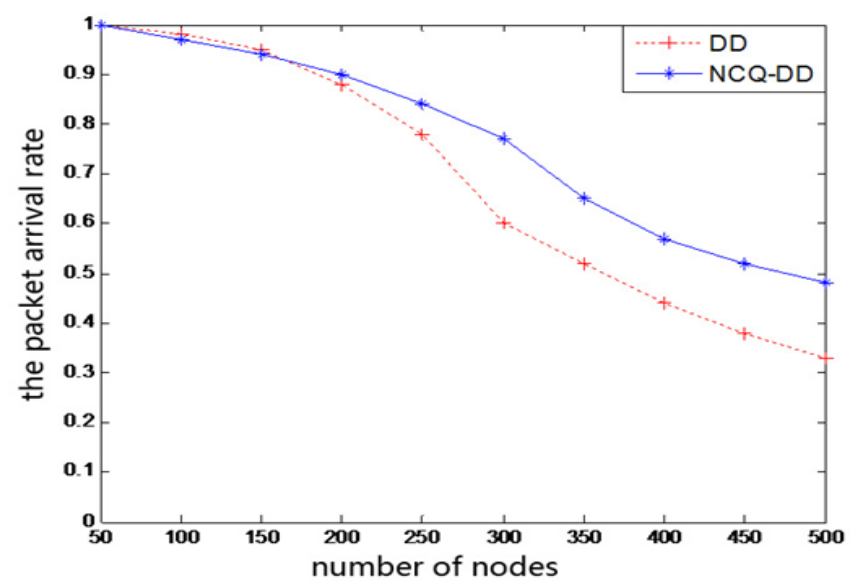

Figure 1. Packet Arrival Rate

From Figure 1, it is apparent that Packet Arrival Rate of these two protocols are both decreased with the increasing number of nodes. In comparison, the rate of DD protocol is declined dramatically and the trend of NCQ-DD protocol is decreased smoothly.

(2) End-to-End Delay

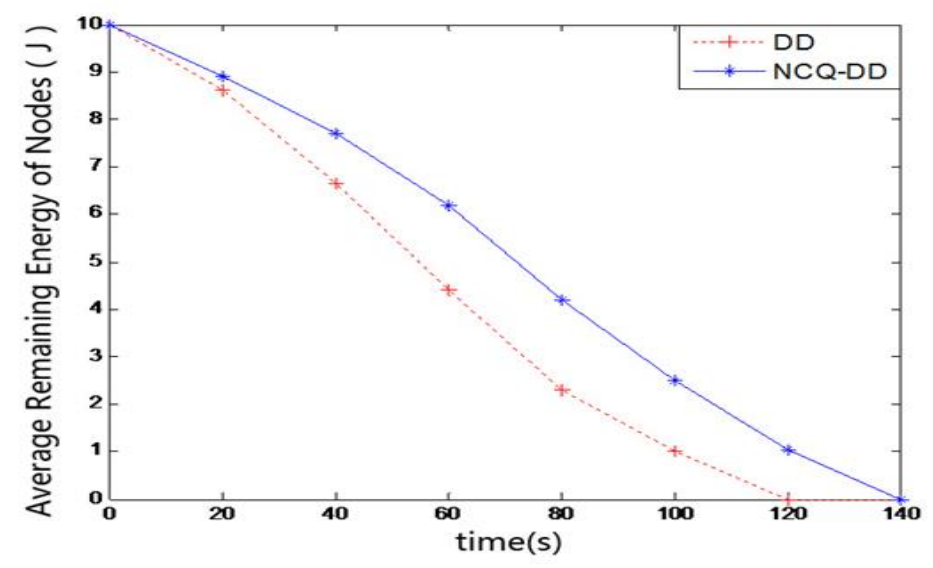

Figure 2. Average End-to-End Delay 
In the Figure 2, the average end-to-end delays of these two protocols are compared. There are several different scenes based on different number of nodes from 50 to 500 divided into 10 sets in this experiment. To each set the average end-to-end delay is recorded and the reliability of network is 0.8. Overall, with the increase of nodes, the average end-to-end delay becomes rising trend for both protocols. However, this rise for DD protocol is more rapid than that for NCQ-DD protocol. It is apparent that with the increasing nodes, the gap of these two lines becomes much wider.

\section{(3) Average Remaining Energy of Nodes}

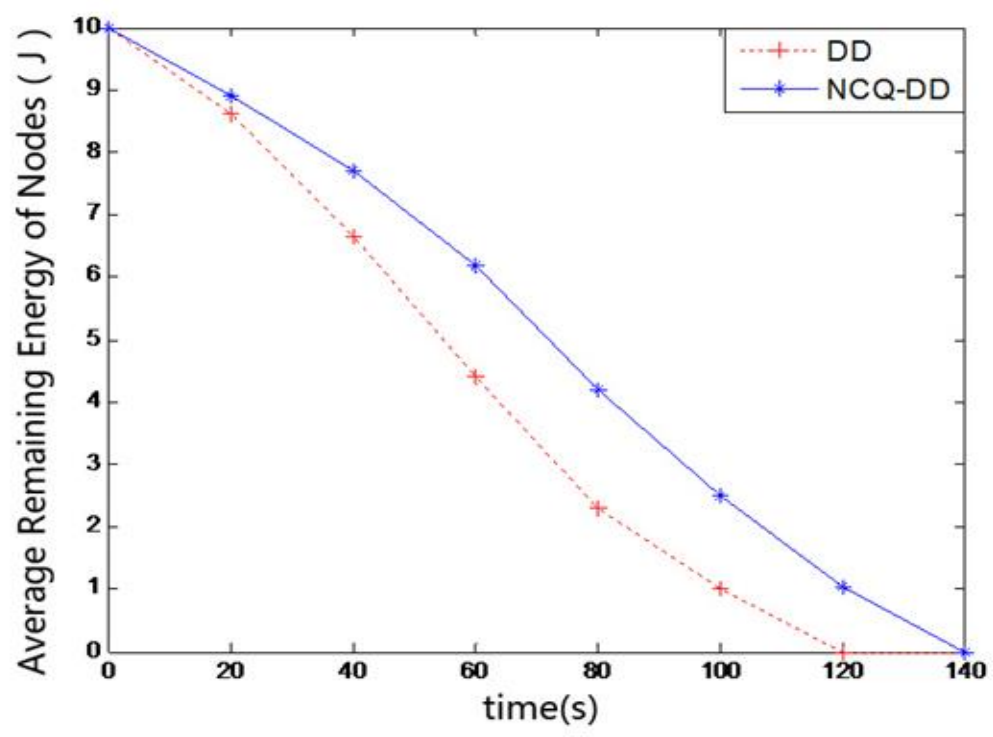

Figure 3. Average Remaining Energy of Nodes

From Figure 3, generally, with the change of time, the remaining energy of nodes for both protocols is gradually declined. Where as in the same time, the remaining energy of nodes for NCQ-DD is better than that for DD. As is shown in this experiment, NCQ-DD protocol is efficient to cut down the energy consumption of network and prolong the life time of network.

\section{Conclusion}

According to encoding the packets by network coding technique, NCQ-DD routing protocol can be efficient to save bandwidth and energy of nodes to improve the efficiency and correctness of data transmission. The delivery rate of packet groups is also improved. Meanwhile, NCQ-DD takes advantage of service awareness mechanism to select routes. It makes full use of the nodes with adequate energy and bandwidth to balance the energy among the nodes, which can reduce the average energy consumption of network and prolong the life time effectively. The results of simulation shows the advantages of NCQ-DD improving network properties in the aspects of energy consumption and transmission delay compared with DD routing protocol.

\section{Acknowledgments}

This research work is supported by the National Natural Science Foundations of P.R. China (NSFC) under Grant (61071093), National 863 Program (2010AA701202), Sweden-Asian International Cooperation Project (348-2008-6212), Jiangsu Province Major Technology Support Program (BE2012849), Jiangsu Province 
industry-university-research prospective joint research project (BY2014014), Jiangsu Province Graduate Research Innovation Project No.CXZZ13_0476 and No.KYLX_0812.

\section{Reference}

[1] S. Yu, B. Zhang and C. Li, "Routing protocols for wireless sensor networks with mobile sinks", a survey, IEEE Communications Magazine, vol. 52, no. 7, (2014).

[2] J. Kulik, W. R. Heinzelman, H. Balakrishnan, "Negotiation based protocols for disseminating information in wireless sensor networks", Wireless Networks, vol. 8, no. 2, (2002).

[3] C. Intanagonwiwat, R. Govindan, D. Estrin and J. Heidemann, "Directed diffusion for wireless sensor networking", IEEE/ACM Trans. on Networking, vol. 11, no. 2, (2003).

[4] B. Chen, K. Jamieson, H. Balakrishnan and R. Morris, "Span: An energy-efficient coordination algorithm for topology maintenance in ad hoc wireless networks", ACM Wireless Networks Journal, vol. 8, no. 5, (2002).

[5] Y Yu, R. Govindan and D Estrin, "Geographical and energy aware routing: A recursive data dissemination protocol for wireless sensor network", UCLA Computer Science Department, Tech Rep: UCLA/CSD-TR-01-0023,(2001).

[6] H. Peng, L. Tang and H. Tang, "Improvement of DD routing protocol in wireless sensor networks", Computer Engineering and Applications, vol. 43, no. 14, (2007).

[7] H. W, Chandrakasan and A, Balakrishnan, "Energy-efficient communication protocol for wireless micro sensor networks", Proc. of the 33rd Annual Hawaii Int'l Conf. on System Sciences, (2000) January, pp. 4-7, Maui, Hawaii, USA.

[8] A. Manjeshwar and D. P. Agrawal, "TEEN: A protocol for enhanced efficiency in wireless sensor networks, Int'l Proc. of the 15th Parallel and Distributed Processing Symp, (2001) April, pp. 23-27, San Francisco, California, USA.

[9] S. Lindsey, C. S. Raghavendra, "PEGASIS: Power-efficient gathering in sensor information systems" Proc. of the IEEE Aerospace Conf, (2002) March, pp. 9-16, Montana ,USA.

[10] D. Braginsky and D. Estrin, "Rumor routing algorithm for sensor networks", Proc. of the 1st workshop on sensor networks and applications, (2002) September, p. 28, Atlanta, Georgia, USA.

[11] Y. Xu, J. Heidemann, D. Estrin, "Geography-informed energy conservation for ad hoc routing”, Proc. of the 7th Annual ACM/IEEE Int'l Conf. on Mobile Computing and Networking, (2001) July, pp. 16-2,1 Rome, Italy.

[12] A. Younism, "An energy-aware QoS routing protocol for wireless sensor networks", the 23rd International Conference on Distributed Computing Systems Workshops, (2003) May, pp. 19-22, Providence, RI, USA.

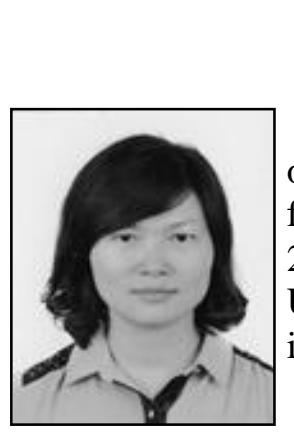

\section{Authors}

Min Zhu, she received her B.S. degree from Nanjing University of Posts and Telecommunications, China in 2002 and M.S. degree from Beijing University of Posts and Telecommunications, China in 2005. Now, she is working toward the Ph.D. degree in Nanjing University of Posts and Telecommunications, China. Her research interests mainly include routing protocol and algorithm design.

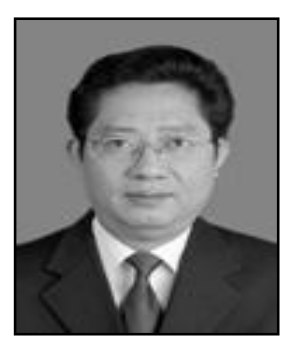

Dengyin Zhang, he received the B.S. degree in telecommunication engineering, the M.Sc. degree in Circuit, Signal and System, and the PhD degree in Signal and Information Processing from Nanjing University of Posts and Telecommunications, Nanjing, China, in 1986, 1989, and 2004, respectively.. He was elected as a master tutor and doctoral tutor in 2000 and 2010 respectively. His main research interest is in the field of signal and information processing, networking technique, Multicarrier (MC), Voice over IP (VoIP), and information security. 


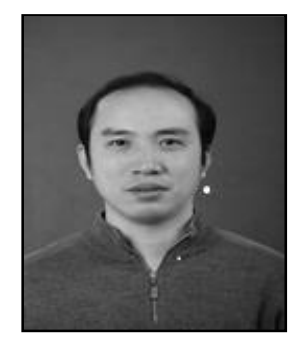

Zhanxiang Ye, he received his B.S. degree from Hangzhou University, China in 1992 and M.S. degree from Wuhan University, China in 2006. His research interests mainly include network and system security.

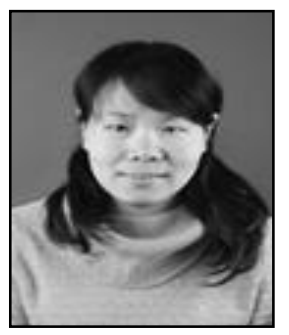

Xuemei Wang she received her B.S. degree from Changchun College of Posts and Telecommunications, China in 2000 and M.S. degree from Nanjing University of Posts and Telecommunications, China in 2003. Now, she is working toward the Ph.D. degree in Nanjing University of Posts and Telecommunications, China. Her research interests mainly include computer vision, computer networks.

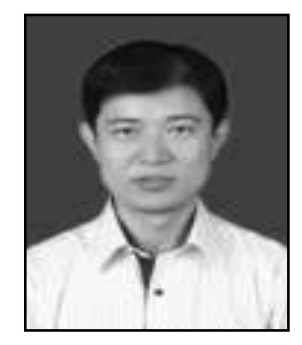

Jin Wang he received his B.S. and M.S. degree from Nanjing University of Posts and Telecommunications, China in 2002 and 2005, respectively. He received Ph.D. degree from Kyung Hee University Korea in 2010. Now, he is a professor in the College of Information Engineering, Yangzhou University. His research interests mainly include routing protocol and algorithm design, network performance evaluation and optimization for wireless ad hoc and sensor networks. He is a member of the IEEE and ACM. 